\title{
Particle swarm-based olfactory guided search
}

\author{
Lino Marques • Urbano Nunes • A. T. de Almeida
}

Published online: 26 May 2006

C) Springer Science + Business Media, LLC 2006

\begin{abstract}
This article presents a new algorithm for searching odour sources across large search spaces with groups of mobile robots. The proposed algorithm is inspired in the particle swarm optimization (PSO) method. In this method, the search space is sampled by dynamic particles that use their knowledge about the previous sampled space and share this knowledge with other neighbour searching particles allowing the emergence of efficient local searching behaviours. In this case, chemical searching cues about the potential existence of upwind odour sources are exchanged. By default, the agents tend to avoid each other, leading to the emergence of exploration behaviours when no chemical cue exists in the neighbourhood. This behaviour improves the global searching performance.

The article explains the relevance of searching odour sources with autonomous agents and identifies the main difficulties for solving this problem. A major difficulty is related with the chaotic nature of the odour transport in the atmosphere due to turbulent phenomena. The characteristics of this problem are described in detail and a simulation framework for testing and analysing different odour searching algorithms was constructed. The proposed PSO-based searching algorithm and modified versions of gradient-based searching and biased random walk-based searching strategies were tested in different environmental conditions and
\end{abstract}

L. Marques $(\bowtie) \cdot$ U. Nunes $\cdot$ A. T. de Almeida

Institute of Systems and Robotics, Department of Electrical and

Computer Engineering, University of Coimbra,

3030-290 Coimbra, Portugal

e-mail: lino@isr.uc.pt

U. Nunes

e-mail: urbano@isr.uc.pt

A. T. de Almeida

e-mail: adealmeida@isr.uc.pt the results, showing the effectiveness of the proposed strategy, were analysed and discussed.

Keywords Olfactive search - Cooperative robotics . Particle swarm optimization

\section{Introduction}

Olfaction is a long distance sense, widely used by animals to find mates, detect hidden resources or threats, and to mark paths and territories. The men has been using animals with a well developed sense of smell, like dogs, to search hard to find targets that release a detectable odour (e.g., some illicit substances, landmines and explosives (Furton and Mayers, 2001)). The use of dogs to find persons in search and rescue (SAR) operations or to persecute someone escaping from justice is also a common procedure nowadays (Hepper and Wells, 2005).

The fear of terrorist threats and an increased demand for high levels of security, boosted the research in ubiquitous artificial olfaction systems able to be spread everywhere in order to quickly detect such threats (Yang, 2005; Stix, 2005). When a sensing node possess mobility, instead of staying still, waiting for the target odour to eventually come into contact with its olfaction system, the node can adopt an active behaviour, moving across its workspace, trying to find traces of the target odour. After detecting an odour trace, the searching agent might track in the upwind direction the odour plume until finding the odour source.

\subsection{Previous works}

The odour tracking problem - i.e. the motion of an agent along an odour plume until its source - has already been 
addressed in the past years by several researchers (Ishida et al., 1994; Russell et al., 1995; Marques et al., 2002b; Rutkowski et al., 2004) and several algorithms have been proposed to solve this task, namely: gradient following, rulebased strategies, and several bio-inspired chemotaxis algorithms, like bacterium biased-random walks and silkworm moth spiral surges. All of these works were demonstrated indoors in a very simplified and controlled testing setup, with the robots travelling small distances across a constant airflow. The release rate of the odour sources was usually high enough in order to guarantee that the robots start already from the active area ${ }^{1}$ of the odour source. Marques et al. (2002b) used a complex experimental setup, composed by two different odour sources placed inside an area with forced advection $^{2}$ and some boxes in the middle. This arrangement, guaranteeing a high degree of turbulence and a consequent mixture of the two odours, was prepared to compare the effectiveness of three common odour-tracking strategies by a robot equipped with two electronic nostrils in environments with multiple odours. Although its increased complexity, this setup was still far from the conditions commonly found in real environments. In these environments, the obstacles, the advection, and the mixture of odours and the respective release rate cannot be controlled, so the airflow is variable in amplitude and direction and the release rate can be very small, leading to odour concentrations not detectable by a remote artificial olfaction system.

\subsubsection{Searching odour sources with multiple robots}

The searching of odour sources can be speed up with the utilization of multiple searching robots, but in this case, as happens with any robotics cooperative task, the additional problem of coordinating the agents in an effective way arise.

The cooperative search problem in robotics can be solved with planned or with reactive approaches. In the planned approach the searching environment should be known by the planner, that assigns target exploration areas to each of the agents using eventually the theory of optimal search (Koopman, 1980; Stone, 1989). This approach, used by several researchers in different searching problems (Burgard et al., 2005), presents as a main disadvantage the centralization in the planner with all the problems associated with centralized architectures, namely, the difficulty of adding and removing searching agents dynamically and the total dependence from one unit: the controller. In the reactive approach, the searching algorithms should be embedded in each searching agent. Each agent might exchange information with other neigh-

\footnotetext{
${ }^{1}$ The area where it is statistically possible to detect traces of the odour released from an odour source.

2 Airflow.
}

bours or not. Comparing to the centralized version, reactive systems might not be optimal, but are more robust and easy to scale up, allowing the emergence of distributed searching systems (Gage, 1993).

The searching of one odour source by multiple robots has been simulated by several authors, but to the best of our knowledge, it was only tried by Hayes (2002). This author implemented a collaborative spiral surge algorithm with several mobile robots to localize an odour source inside a square arena with about $40 \mathrm{~m}^{2}$. This experiment was also simulated with good agreement between experimental and simulated results.

The usage of a large number of cooperating robots makes sense for searching across very large searching spaces - several orders of magnitude the size of the robots. Searching in such large dimensions places new problems for the agents that are the lack of stimuli inside most of the searching space. This problem is addressed by the theory of optimal search, but not using olfactory sensing ${ }^{3}$ (Koopman, 1980; Stone, 1989). Considering the searching of odour sources near the atmospheric boundary layer, such large spaces are only available in outdoor environments, being hard to group a large number of robots able to move in outdoors for making cooperative odour search experiments. An alternative to a real setup can be a realistic simulated setup for trying and analysing the performance of different searching algorithms. Simulation is an effective tool, particularly to test the behaviour of complex systems that are difficult or not practical to test experimentally in real environments, like the searching of odour sources in large outdoor spaces. Farrell et al. (2003) for example shown the utilization of hidden Markov methods to locate an odour source in a simulated environment. Marques et al. (2002a) used a Genetic Algorithm to coordinate a group of mobile robots searching for an odour source. The proposed algorithm was effective when the agents start in random positions, but was slow to converge when the agents start from a corner of the search space (a more realistic situation). Two other problems of this algorithm were the difficulty to search multiple sources and the impossibility of integrating the wind information. These problems were addressed by a latter work with an Evolutionary-Based search algorithm (Marques et al., 2003c). The main problem that persisted with this algorithm was its centralized nature. This aspect is solved with the Particle Swarm-based approach proposed in this article.

\section{Problem formulation}

When a volatile leaves its source, it is carried by the wind generating what is called an odour plume. An odour plume

\footnotetext{
${ }^{3}$ Vision and radar are the most common sensor types employed.
} 
presents typically a conic shape spreading laterally in the downwind direction. The plume length is mainly determined by advection phenomena and the lateral spreading is mainly due to turbulent diffusion. ${ }^{4}$ As a consequence of this behaviour, the spatial and temporal average odour concentration decrease when the distance to the odour source increases.

Since an odour source located near the ground level release an odour profile with maximum average concentration near the source, the problem of searching multiple such odour sources in a limited environment can be formulated as a stochastic optimization problem where the goal is to find local maxima in the average odour concentration map.

There are two main aspects that should be modelled to simulate the searching of odour sources by mobile robots. The first aspect is the odour transport in the environment and the second aspect is the modelling of the mobile robots (also called searching agents in this article).

\subsection{Odour plumes}

Odour molecules released in natural environments are carried by the wind forming an odour plume. As the plume travels away from the source, it becomes more diluted due to diffusion. Two processes cause the diffusion of odours: molecular diffusion and turbulence. Molecular diffusion is a very slow process whose effect can be neglected for a plume characterization. Turbulent diffusion is manifested in the boundary layer by different size eddies (Yee et al., 1994; Mylne and Mason, 1991; Mole and Jones, 1994). Small scale eddies shred an odour plume into filaments causing in-plume concentration fluctuations and large scale eddies, bigger than the plume width, cause plume meandering. Small scale eddies are mainly caused by shear stress of the wind on the surfaces (mechanical turbulence). Large scale eddies are formed by the heat flux coming from surfaces warmed by the sun (thermal turbulence). The action of eddies on the plume can be separated in three zones. Near the odour source eddies with size much larger than the plume width cause plume meandering. In the second zone it is possible to observe high concentration intermittency periods caused by eddies with diameter identical to the plume diameter. Those eddies shred the plume by introducing puffs of clean air inside the odour patches, producing high peak-to-mean concentration ratios. Far away from the odour source, the turbulence act like mixers that homogenize the plume. In this zone the instantaneous concentration is mainly small and uniform, but even so it is possible to detect some fast concentration peaks.

The lack of smooth concentration gradients found in real outdoor atmospheric environments means that a simple gra-

\footnotetext{
${ }^{4}$ Other phenomena, like molecular diffusion and chemical reactions also exist, but are not relevant in the space and time scales of an odour searching problem.
}

dient following strategy cannot be used to find an odour source. Far enough downwind of the source, odour plumes are filamentous and sporadic. The instantaneous concentration measured by a fast chemical sensor placed downwind the source will fluctuate with large intermittency periods and short concentration peaks that can be well above three orders of magnitude the average concentration value (Mylne and Mason, 1991). Under these circumstances, gradient-based navigation with an array of scattered gas sensors will be impracticable because the time-averaged local gradient is too small and the instantaneous gradient is time varying, steep and random-like. In this case, more complex strategies are required and additional information, such as airflow velocity and direction are essential (Balkovsky and Shraiman, 2002).

In previous works, meandering odour plumes were simulated with CofinBox ${ }^{5}$ software package. This program allows to specify different environmental conditions concerning terrain type, gas released, wind characteristics, time and downwind distance and outputs to a text file the plume centre $y_{0}(x, t)$, width $w(x, t)$ and height $h(x)$ as a function of time $t$ and downwind distance $x$. The instantaneous odour concentration of a meandering plume, at ground level $(z=0)$, can be determined by the following Gaussian expression:

$$
C(x, y, t)=\frac{Q}{2 \pi \sigma_{y}(x, t) \sigma_{z}(x)} \exp \left(-\frac{\left(y(t)-y_{0}(x, t)\right)^{2}}{2 \sigma_{y}^{2}(x, t)}\right)
$$

with $Q$ representing the release rate, $\sigma_{y}(x, t)=w(x, t) / \sqrt{2 \pi}$ and $\sigma_{z}(x)=h(x) / \sqrt{2 \pi}$. Figures 1 and 2 show an example of a time averaged Gaussian plume and the shape of an instantaneous meandering plume.

CofinBox did not simulate internal plume intermittency. In the current work this limitation is overcome integrating the models proposed by COFIN project in Matlab scripts along with the intermittency model proposed by Anderson et al. (2001). This intermittency model uses a Markovmodulated Poisson process (MMPP) to model the rate $\lambda_{i, k}$ of occurrence of large concentration values inside an odour plume:

$\lambda_{i, k}=\rho \cdot \exp \left\{-\frac{\left(r_{k}-y_{i}\right)^{2}}{2 \sigma_{y}^{2}}\right\}$

where $\sigma_{y}$ is a measure of the instantaneous plume width, and $\rho$ is the rate at which large concentration occur at the plume centreline and $r_{k}$ is the receptor distance from the plume centreline $y_{\mathrm{i}}$.

\footnotetext{
${ }^{5}$ This package was developed in the framework of European Union COFIN project (Nielsen et al. 2002).
} 


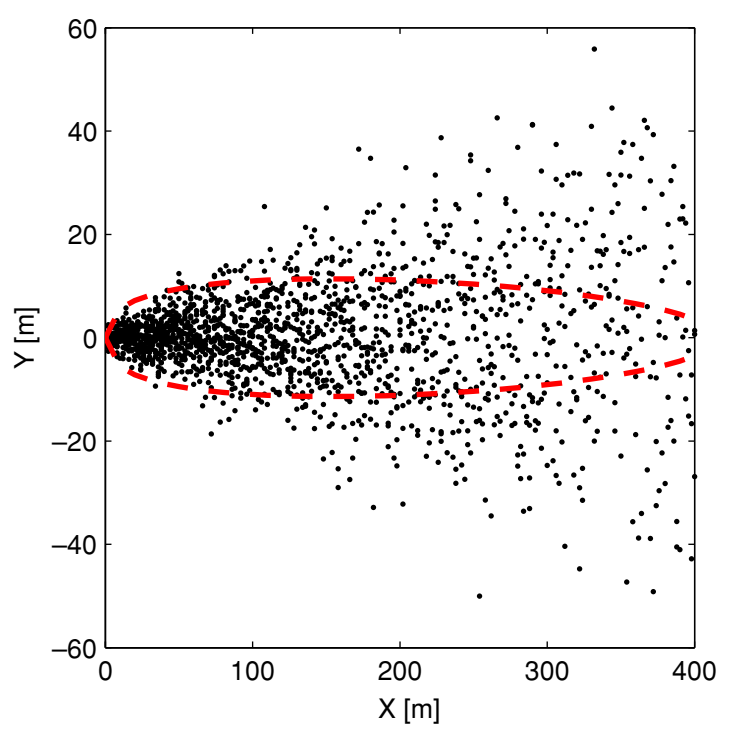

Fig. 1 Concentration at the ground level of a time averaged Gaussian plume generated from a continuous point source localized at ground level in a planar surface. Note that the axis in the figure are not in the same scale

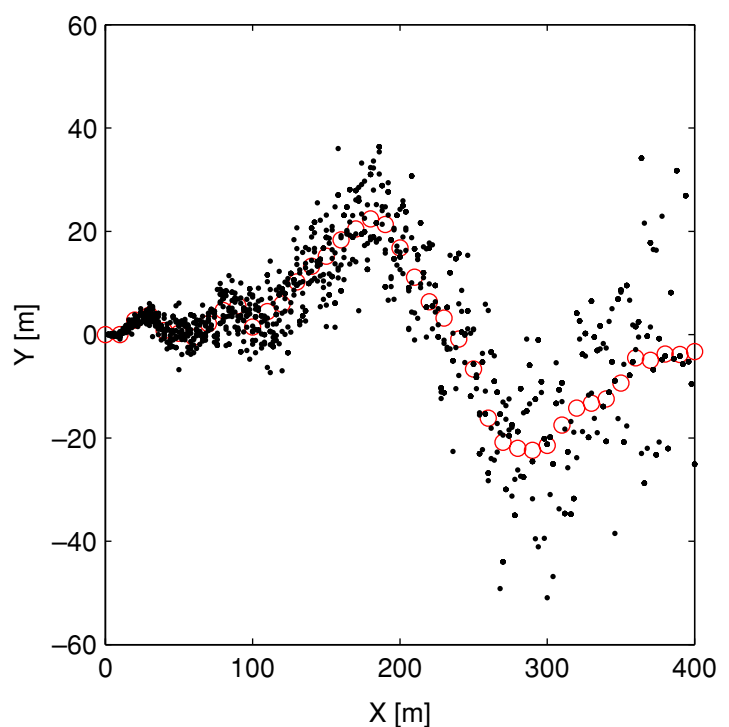

Fig. 2 Instantaneous centerline and width of a meandering chemical plume

\subsection{Searching agents}

There are two main aspects that need to be considered about odour sources searching agents: their mobility and their ability to sense odours.

\subsubsection{Olfaction systems}

From the above description, it becomes clear that an olfactory sensing system for tracking odour plumes until their source should have the following characteristics:
Listing 1. Agent's motion till a target position.
While Not_In_Target Do
Calculate Attraction force to the target
Calculate Repulsion force from other agents
Calculate Repulsion force from static obstacles
Update velocity
Update position
End

- It should be fast in order to detect small odour patches far away from the source.

- It should be selective in order to discriminate the odour searched from other similar odours that might be present in the environment.

- It should sense airflow intensity and direction in order to perceive where the odour comes from and what atmospheric conditions exist.

There are very fast chemical sensing systems, e.g., photoionization detectors (PID), which are sometimes employed in the study of the internal structure of odour plumes (Justus et al., 2002), but usually those systems are not selective. The metal oxide chemical sensor (MOS) is the sensing device more frequently employed for odour plume tracking. For example, in Marques et al. (2003b) and in Almeida et al. (2004) was presented an olfactory sensing system for mobile robotics composed by a directional anemometer and a smart sensing nostril (see Fig. 3). The thermal anemometer measures airflow intensity and direction using four selfheated thermistors placed around a square wind deflecting pillar. The power dissipated by each sensor depends from the difference between the device temperature and local air temperature and from the airflow around the device. The typical orientation error of this anemometer is in the range of 5 to 10 degrees. The smart gas sensing nostrils use an array of four different metal oxide gas sensors operated in temperature-modulated mode by a microcontroller-based signal conditioning circuit allowing to implement a large sensing space electronic nose (Marques et al., 2003b). The selectivity and fast identification of the air mixtures is assured by the type of signal processing employed to the gas sensing array output, namely discrete wavelet transform (DWT) for transient analysis and principal component analysis (PCA) and artificial neural networks (ANN) for pattern recognition (Almeida et al., 2003). This system presented a response time in the order of 2 seconds and a sensitivity that depends from the gases, but for common ethanol it was below $1 \mathrm{ppm} .^{6}$ Figure 4 exemplifies the effect of sampling a fast intermittent plume with a limited response-time chemical sensing system.

\footnotetext{
${ }^{6} \mathrm{ppm}$ - parts per million.
} 
Fig. 3 Front and back pictures of a gas sensing nostril
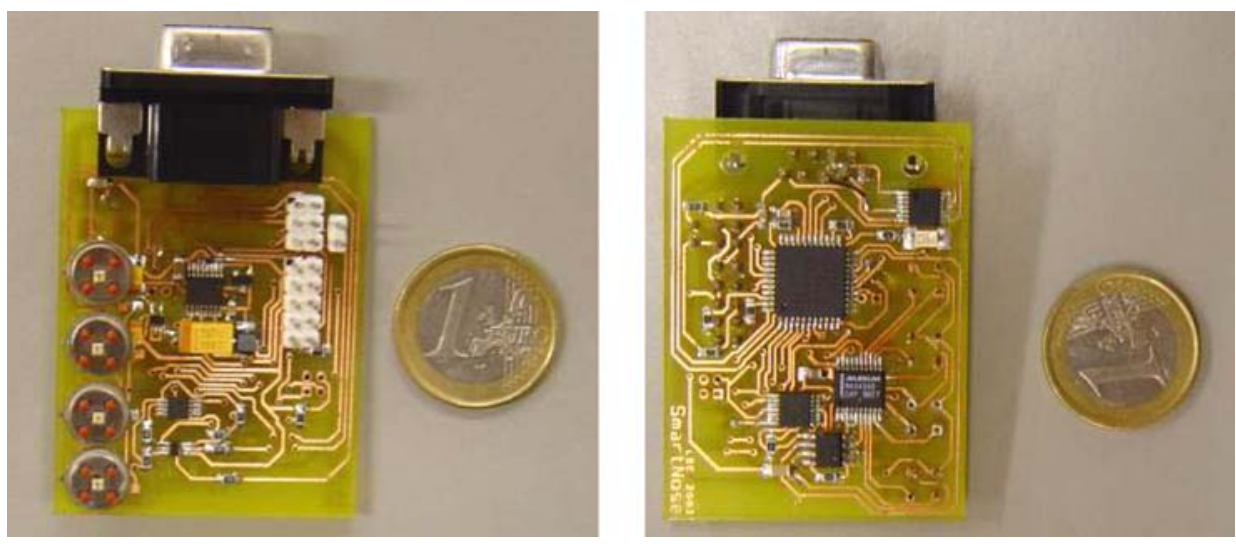

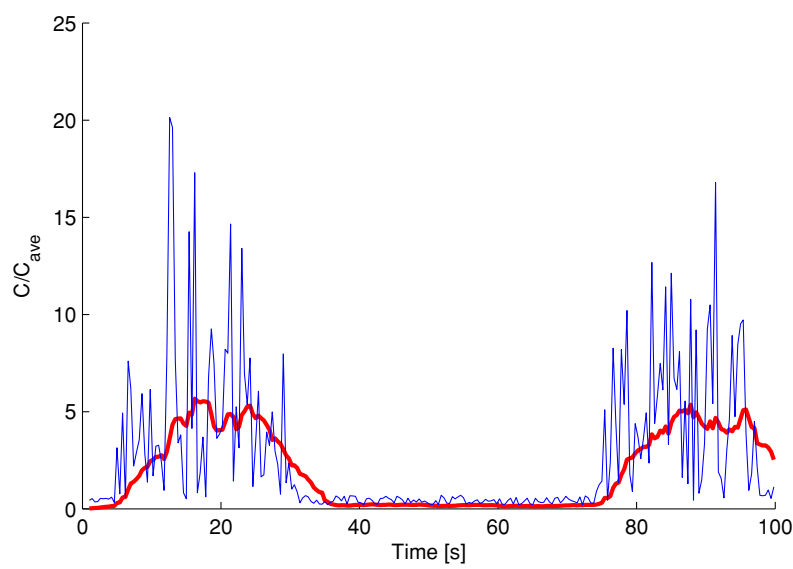

Fig. 4 Instantaneous concentration measured by a very fast chemical sensor placed in the active area of an odour plume. An electronic nose like the one described shows a response time of about $2 \mathrm{sec}-$ onds, providing a corresponding low-pass filtered output to the signal represented

\subsubsection{Motion control}

The searching agents can do self motivated motions, constrained in terms of maximum velocity and maximum acceleration. The agents are assumed to be able to localize themselves inside the workspace and localize the obstacles in the neighbourhood. The workspace contains a variable density of obstacles. The motion of the agents is commanded by means of a potential field-based method (Latombe, 1991) with target points representing attractive potentials and obstacles and other agents repulsive ones (see Listing 1). In order to solve potential minima, each time a target is reached or an agent velocity becomes too small, a new target position will be generated by the searching algorithm.

\subsection{Search parameters}

The main parameters considered in this work for a searching experiment are environment related parameters:
- dimensions of the workspace - the workspace is considered rectangular with Length $\times$ Width square meters. The spatial coordinates are referenced to the bottom left corner;

- obstacles - all obstacles are considered circular with variable radius. The parameters of each obstacle are the localization of its centre and its radius $\left\{x_{0}, y_{0}, r_{0}\right\}$;

- odour sources - each odour source $s$ is characterized by its localization and release rate $\left\{x_{\mathrm{s}}, y_{\mathrm{s}}, Q_{\mathrm{s}}\right\}$;

- wind - the wind is characterized by its average velocity and the variances in intensity and direction $\left\{u, \sigma_{u}, \sigma_{\theta}\right\}$, for simplicity, the average wind direction is always considered aligned with one of the workspace axis (in this case the $Y Y$ axis);

and agents' related parameters:

- the quantity of agents, their dynamic properties, and the communication range $\left\{N r o b, v_{\max }, a_{\max }, r_{\text {comm }}\right\}$;

- the sensitivity and time response of the olfaction systems employed $\left\{C_{t h}, \tau_{c}\right\}$;

- the radius-of-detection of the odour source identification system;

- the initial status of each agent $\left\{x_{r}, y_{r}, \theta_{r}, v_{r}\right\}$;

- the simulation period $T$;

- and finally, the searching algorithm employed $\{P S O$, $B R W, G R D\}$ and their respective parameters (e.g., the maximum step $d$ for a biased random walk-see Section 3).

\section{Collective searching}

A typical problem of search algorithms is the conciliation between the ability of an algorithm to exploit new search cues and its ability to explore the whole search space looking for new cues. For example, common reactive search algorithms, like gradient climbing are effective in finding the peak of a bell-shaped field, but cannot be used to search odours across large spaces because in most of the space no concentration 


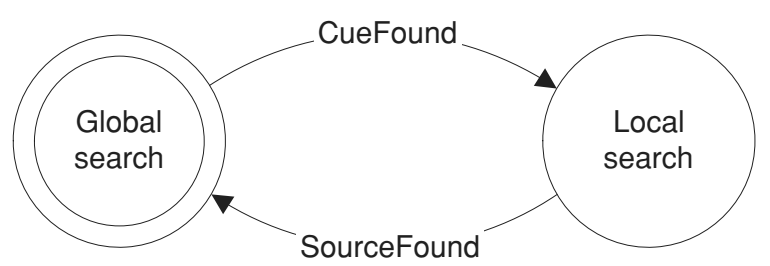

Fig. 5 State diagram representing the switching between global and local-searching modes

can be sensed and so the agents become stopped without exploring unknown areas.

Both the exploration of new areas and the exploitation of chemical cues eventually found can be solved by means of a state-based search strategy that changes the strategy from global search (exploration) to local search (exploitation) whenever a new search cue was found (Marques et al., 2003c) (see Fig. 5). This intermittent behaviour (sometimes called "saltatory") was also observed in several animals while hunting (Bénichou et al., 2005).

\subsection{Local searching}

In the context of searching for odour sources, local searching is related to the phases of chemical plume tracking and source localization. Local searching algorithms are frequently classified between gradient-based algorithms (GRD), when the searching agent moves in the gradient direction and random algorithms, when the searching agent don't care about the sensed values. Biased random walks (BRW) and Evolutionary strategies (ES) are stochastic methods that can be classified in between the previous ones. The stochastic word means here that there are always some random components in the searching movements.

BRW keeps the average direction of search while the sensed value improves. If that value decreases, the agent turns the motion to a completely random direction (Müller et al., 2002; Passino, 2002). This algorithm, inspired by the motion of bacteria, even for very noisy fields tends to search in the gradient direction, but can be very slow to converge (find the goal). Sections 3.1.1 and 3.1.2 explain the Gradientbased and BRW-based algorithms employed in this work.

Evolutionary strategies are based in keeping a population of good solutions and explore preferentially new areas in the direction of those good solutions. Since ES are based in a population of solutions, these methods are very easy to parallelize and adapt to a group of searching agents. As can be easily understood, these methods also tend to move in the gradient direction.

Some moth's zigzagging and surge motions are frequently referred as one of the most successful methods for odour plume tracking in turbulent atmospheres (Balkovsky and Shraiman, 2002). This strategy shares some aspects with
Listing 2. Gradient climbing local searching algorithm.

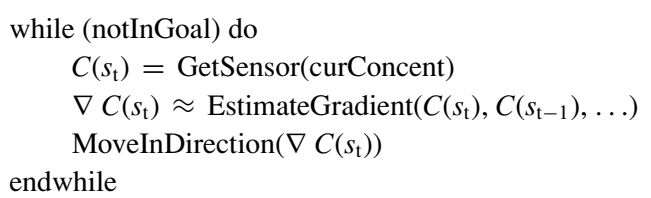

stochastic methods, namely the combination of crosswind and upwind biased random motions across the plume, but this process is difficult to parallelize.

Particle Swarm Optimization (PSO) can be adapted to integrate most of the good aspects of the previous methods. Furthermore, the algorithm can be easily adapted to a community of mobile searching agents exchanging information among them. All local search strategies implemented in this work use the chemical information and an upwind bias to generate new target positions to search.

\subsubsection{Gradient search}

Gradient based methods are known to perform well optimizing smooth (differentiable) and unimodal functions (like the average concentration presented by a Gaussian plume). With this type of function it is enough to follow the gradient direction in order to find the position of maximum value.

$\mathbf{s}_{t+1} \leftarrow \mathbf{s}_{t}+\alpha \cdot \nabla f\left(\mathbf{s}_{t}\right)$

where $\mathbf{s}_{t}$ represents the space coordinates at time $t$, and $\alpha$ is a small positive constant. In the cases where the function $f$ is not analytically known, the gradient can be empirically estimated by evaluating changes $\Delta f$ to small increments in the different space dimensions $\Delta \mathbf{s}$. Listing 2 shows a possible algorithm for following a chemical concentration gradient.

\subsubsection{Biased random walks}

Biased random walks are simpler to implement, since this algorithm only compares the current concentration with the concentration measured in the previous sample. The following local searching BRW algorithm was adapted from Holland and Melhuish (1996) where $d$ corresponds to the maximum displacement desired per sample time.

\subsubsection{The particle swarm optimization algorithm}

The Particle Swarm Optimization algorithm is an evolutionary technique originally proposed by Kennedy and Eberhart (1995). This optimization method takes inspiration from the dynamics of social organisms while searching for food, 
where social sharing of information takes place and individuals can profit from the discoveries and previous experience of all other companions (e.g., flocks of birds and herds of fishes). The original PSO algorithm assumed the existence of a group (or swarm) of searching elements (called particles)

Listing 3. E. Coli bacteria's BRW chemotaxis algorithm

while (notInGoal) do

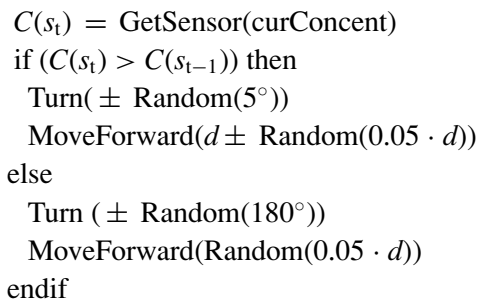

that move across a $D$-dimensional search space according to the following rules:

$$
\begin{aligned}
v_{i}(t)= & \phi v_{i}(t-1)+\rho_{1}\left(x_{\text {pbest } i}-x_{i}(t)\right) \\
& +\rho_{2}\left(x_{\text {gbest }}-x_{i}(t)\right), \\
x_{i}(t)= & x_{i}(t-1)+v_{i}(t),
\end{aligned}
$$

where $v_{i}$ and $x_{i}$ represent the $i$ th particle's velocity and position vectors respectively; $x_{\text {pbest } i}$ and $x_{\text {gbest }}$ represent the particle previous best value and a predefined neighbourhood best value respectively; $\phi$ is a constriction factor that allows to control the magnitude of the velocity; and $\rho_{1}$ and $\rho_{2}$ are two positive random values. The first equation updates the particle's velocity taking into account their previous velocity and the distances to the particle's best previous position and to the swarm's best experience. The second equation updates the particle's position by adding the calculated velocity to the particle's previous position. The $\phi$ value controls the explorative behaviour of the algorithm (higher $\phi$ values impose a behaviour more explorative). The ratio between $\rho_{1}$ and $\rho_{2}$ controls how collective and self experience influence future searching directions. More recent approaches of this technique change dynamically the magnitude of these coefficients, allowing to adapt the searching behaviour of the algorithm to the characteristics of the problem in hand (Parsopoulos and Vrabatis, 2002).

\subsubsection{PSO-based robotic searching}

To search odour sources with robotic agents using a PSObased algorithm, the agents should be able to localize themselves, measure local odour concentrations and keep the position of higher concentration and share this position with other neighbour agents. The PSO-based searching algorithm
Listing 4. PSO-based searching algorithm.

Initialize the population

Do

Evaluate agent's fitness

Compare each agent's fitness

Calculate target positions using PSO

Move till the target

Until finishing_criterium

represented in Listing 4 can use each agent's time-averaged concentration value as the fitness function in order to generate new target search positions. An odour source can be found by circumnavigating a suspicious area with closing spirals (Marques et al., 2003a) or using a second detection mechanism to identify the source when an agent passes sufficiently near (e.g., a vision system for a visible source). In this paper this last case is considered supposing the utilization of sensors with $1 \mathrm{~m}$ radius-of-detection. Each source found becomes collected and its respective odour field disappear. The searching process can stop after a pre-specified time or after a pre-specified number of sources have been found.

\subsection{Global searching}

Global searching is employed when an agent does not have a sensing cue to exploit, so the best thing to do is to explore the environment trying to find such cue (see Fig. 5). As was previously described, odour plumes are elongated by the wind, so, crosswind movements maximize the detection width of the olfaction system. As the wind becomes more unstable, the plume becomes less elongated and wider. In this case, the benefits of crosswind trajectories disappear.

\subsubsection{Initialization of the population}

Most population based search and optimization algorithms found in the literature start the population randomly across the search space. Whilst this type of initialization provides a good sampling of the space from the beginning, it is not very realistic to implement in robotic search agents, since usually those agents start searching from the same area, usually a corner of the search space.

A global searching behaviour integrating repulsive forces between the agents and crosswind biased motion is particularly important in this phase, since it guarantees a quasioptimal spreading of the agents across the whole search space. Each time an agent finds a chemical cue, it exploits the cue by means of a local searching algorithm (e.g., PSObased; Gradient Ascent; and chemical concentration Biased Random Walk). 


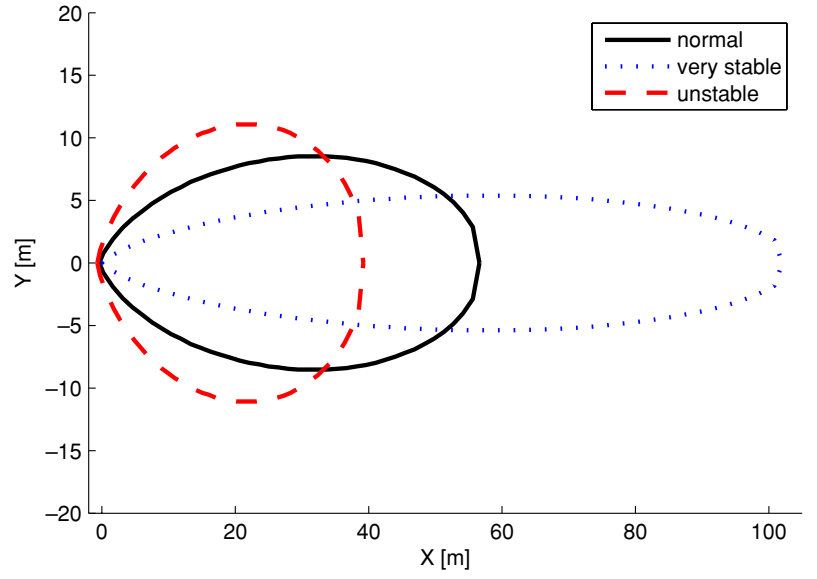

Fig. 6 Active area of the three test plumes used in the simulations. An odour source searching agent will have maximum detection width for crosswind movements in stable atmospheres (longer odour plumes).

\section{Simulation experiments}

Simulation experiments based in appropriate physical models are a convenient mean to explore parameter space in a way that is not possible with real experiments. In simulation the searching parameters can be varied one at a time in order to test different combinations of search environments and agents' properties, allowing in this case to compare the relative performance of the proposed PSO-based algorithm with other "standard" searching algorithms.

\subsection{Searching benchmarks}

The performance of the different searching algorithms should be assessed against an adequate performance index. Some performance indexes commonly employed are the average time taken or the distance ${ }^{7}$ travelled to find a number of odour sources in a specified environment. Another possible performance index can be the robustness, i.e. the certainty to find all odour sources in a fixed period of time. This work employed the searching time as the performance index.

\subsection{Simulation setup}

Considering the enormous amount of parameters that can be changed and the stochastic nature of each experiment, some of the parameters, considered less important for the main goal of this work, were kept constant.

The workspace dimension was set to $200 \times 200 \mathrm{~m}^{2}$ and the sampling time was set to $2 s$. This workspace was pop-

\footnotetext{
${ }^{7}$ For constant velocity experiments, searching time is proportional to the distance travelled. Since in this case the velocity of the agents is controlled by potential fields, the two indexes are not the same.
}

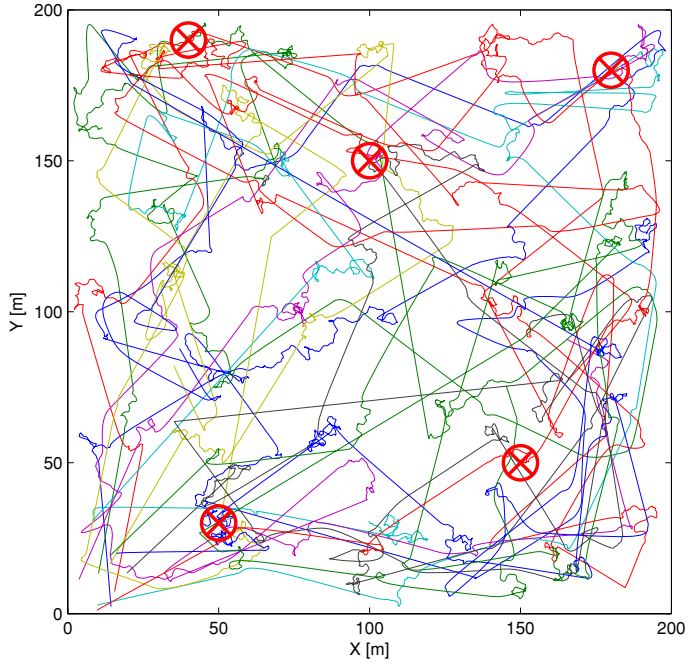

(a) BRW local searching

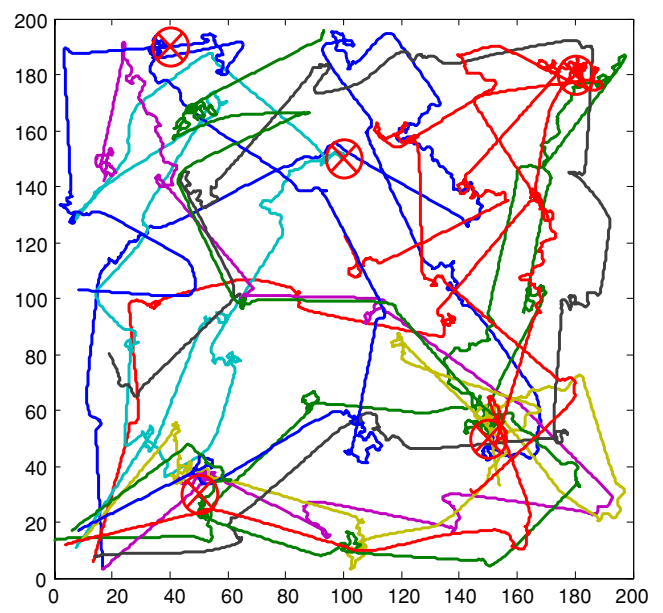

(b) Gradient following

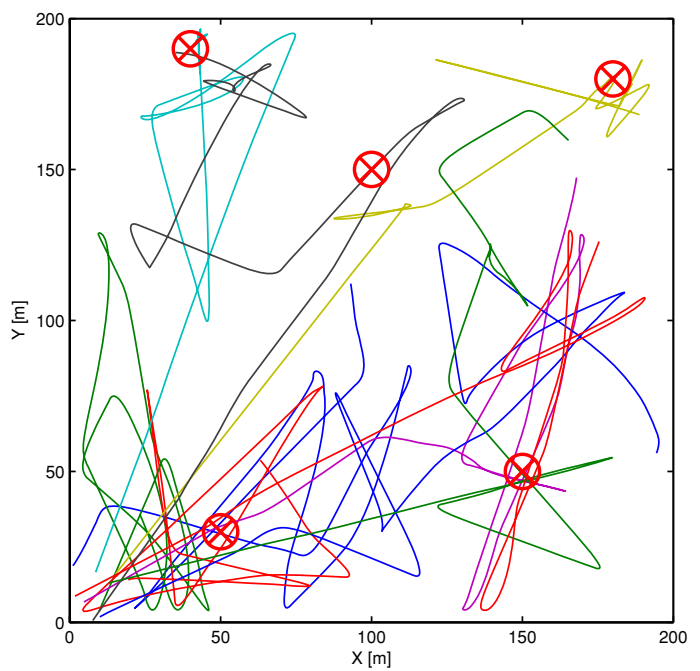

(c) PSO local searching.

Fig. 7 Example with the trajectories described by a swarm of 10 robots searching for the source of meandering odour plumes controlled by different local searching strategies 


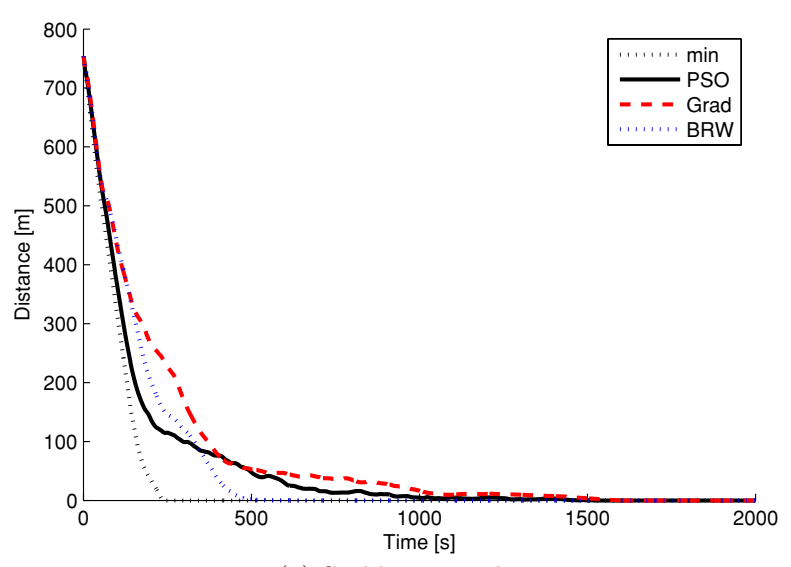

(a) Stable atmosphere

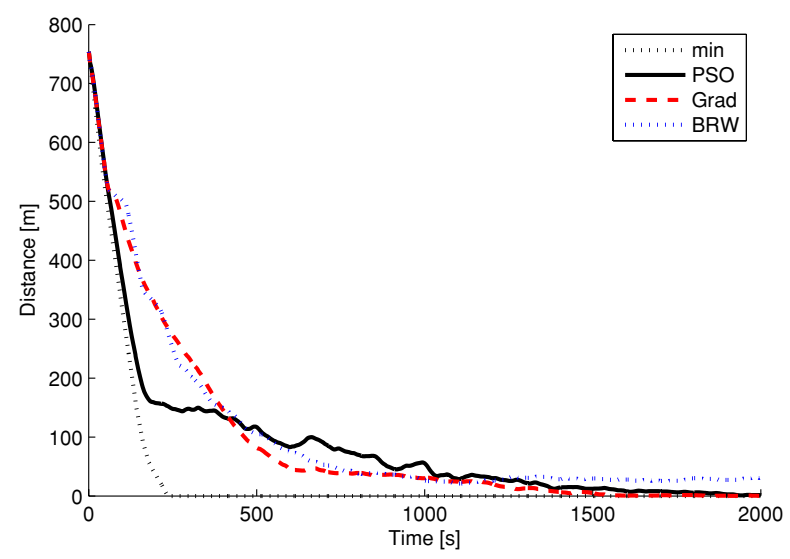

(b) Normal atmosphere

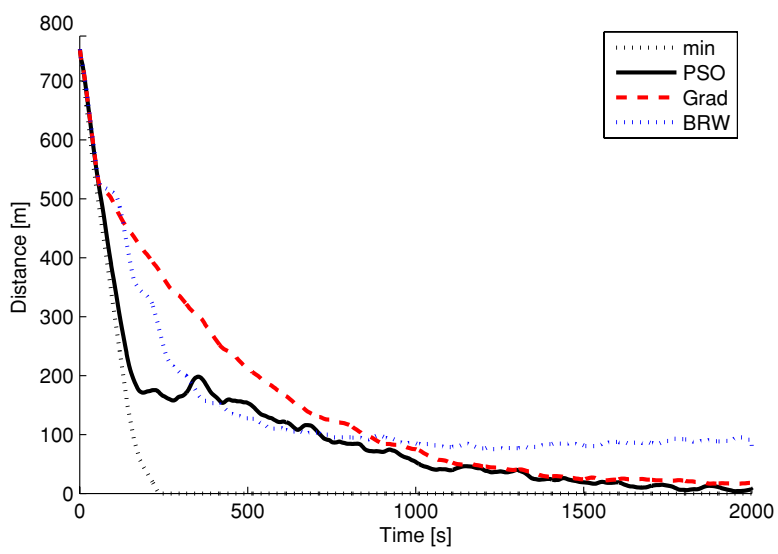

(c) Unstable atmosphere

Fig. 8 Statistical analysis of the average distance to the odour sources in the simulation environment for the three different stability classes.

ulated with a variable number of obstacles, representing an occupied density of $0,5,10$ and $20 \%$ of the workspace area. The motion parameters of the agents was kept constant with maximum velocity and acceleration bounded to $1 \mathrm{~m} / \mathrm{s}$ and $0.1 \mathrm{~m} / \mathrm{s}^{2}$ respectively. The number of sources and their release rate was kept constant $\left(N_{s r c}=5\right.$ and $\left.Q=1 \mathrm{~g} / \mathrm{s}\right)$. The sensitivity of the olfaction systems was set in order to detect a normal odour plume in its centre at $50 \mathrm{~m}$ from the

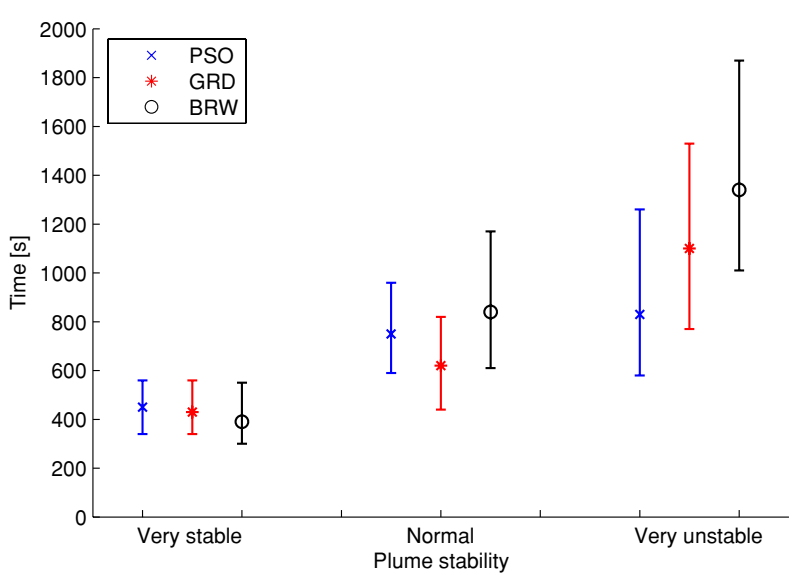

Fig. 9 Statistical analysis of the average time spent to find three odour sources in the simulation environment

source with a $P O D=0.1 .^{8}$ The wind velocity and fluctuation parameters were changed in order to obtain three types of plumes: normal, elongated, and wide (see in Figure 6 the active area of each plume for $Q$ and $C_{\text {th }}$ considered).

\subsection{Simulation results}

As expected, since the average velocity decreases when the obstacle density increases, the searching performance decreased with the density of obstacles, but it was not found a correlation between the obstacle density and the comparative performance of the three algorithms in test, so the study comparing the three algorithms considered always the same obstacles occupying about $5 \%$ of the workspace.

The main focus of this work was testing the performance of the proposed PSO-based algorithm in the presence of atmospheres with different degrees of turbulence. Figure 7(a) to (c) shows example trajectories of BRW, GRD and PSObased searching experiments described by 10 agents searching for 5 odour sources. These simulations were performed in environments with normal plumes.

The three searching algorithms were run 20 times each for each of the previously described environmental conditions. An interesting indicator about the performance of each algorithm during the search is shown by Figure 8(a) to (c). This Figure shows for each atmosphere, the average cumulative minimum distance to the odour sources to be found (global proximity of the searching agents to the odour sources). The dotted line in those Figures represents the best possible performance, i.e., the evolution of the indicator if the agents knew in advance where the sources were localized. The analysis of the Figures demonstrates comparatively good running results for the PSO-based searching algorithm.

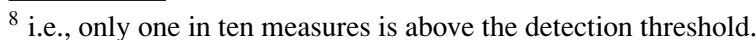


Figure 9 shows the average time taken to find the first three odour sources. From this figure it is possible to see that while PSO-based search was the worst algorithm for the stable atmosphere, it becomes better when the turbulence level increases and it is clearly the best search algorithm for the most unstable atmosphere considered.

\section{Conclusions}

The current research about searching odour sources has been concentrated in the problem of tracking odour plumes. Although important, this problem is only one part of the whole olfaction-based searching, because before tracking a plume, it is necessary to find it.

This work proposed a cooperative algorithm, based in the exchange of information between neighbouring searching agents that performed favourably relatively to gradient search and to biased-random walk search.

The comparative experiment was made in a simulation environment able to simulate the transport of odours in large outdoor spaces. The models employed in this environment were described. Some parameters of the search algorithms were attributed heuristically. ${ }^{9}$ In future works, the authors intend to optimize those parameters and validate the algorithms searching odour sources in a large real testing setup.

\section{References}

Almeida, N., Marques, L., and de Almeida, A. 2003. Fast identification of gas mixtures through the processing of transient responses of an electronic nose. In Proc. of EuroSensors.

Almeida, N., Marques, L., and de Almeida, A. 2004. Directional electronic nose setup-results on the detection of static odour sources. In IEEE Int. Conf. on Sensors.

Anderson, C., Mole, N., Nadarajah, S., and Rydén, T. 2001. Modelling the occurrence of large concentration values in pollutant plumes. In Proc. Int. Cong. on Modelling and Simulation, pp. 911-916.

Balkovsky, E. and Shraiman, B. 2002. Olfactory search at high Reynolds number. Proc National Academy of Science USA, 99(20):12589-12593.

Bénichou, O., Coppey, M., Moreau, M., Suet, P.H., and Voituriez, R. 2005. A stochastic model for intermittent search strategies. Journal of Physics: Condensed Matter, 17(49):S4275-S4286.

Burgard, W., Moors, M., Stachniss, C., and Schneider, F. 2005. coordinated multi- robot exploration. IEEE Transactions on Robotics, 21(3):376-386.

Farrell, J.A., Pang, S., and Li, W. 2003. Plume mapping via hidden markov methods. IEEE Trans. on Systems, Man, and Cybernetics - Part B, 33:850-863.

Furton, K. and Myers, L. 2001. The scientific foundation and efficacy of the use of canines as chemical detectors for explosives. Talanta, 54(3):487-500.

\footnotetext{
${ }^{9}$ For instance, the step distances in BRW and GRD.
}

Gage, D. 1993. Randomized Search Strategies with Imperfect Sensors. In Proc. SPIE Conf. on Mobile Robots VIII: 270-279.

Hayes, A.T. 2002. Self-organized robotic system design and autonomous odor localization. Ph.D. thesis, California Institute of Technology.

Hepper, P. and Wells, D. 2005. How many footsteps do dogs need to determine the direction of an odour trail? Chemical Senses, 30(4):291-298.

Holland, O. and Melhuish, C. 1996. Some Adaptive movements of animats with single symmetrical sensors. In Proc. 4th Conf. on Simulation and Adaptive Behavior-From Animals to Animats, 4. pp. 55-64.

Ishida, H., Suetsugu, K., Nakamoto, T., and Moriizumi, T. 1994. Study of autonomous mobile sensing system for localization of odor source using gas sensors and anemometric sensors. Sensors and Actuators, A45:153-157.

Justus, K., Murlis, J., Jones, C., and Cardé, R. 2002. Measurement of odor-plume structure in a wind tunnel using a photoionization detector and a tracer gas. Environ. Fluid Mech, 2:115-142.

Kennedy, J. and Eberhart, R.C. 1995. Particle swarm optimization. In IEEE Int. Conf. on Neural Networks, pp. 1942-1948.

Koopman, B. 1980. Search and Screening: General Principles with Historical Applications. Pergamon Press.

Latombe, J. 1991. Robot Motion Planning, Kluwer.

Marques, L., Almeida, N., and de Almeida, A. 2003a. Mobile robot olfactory sensory system. In Proc. of EuroSensors.

Marques, L., Almeida, N., and de Almeida, A. 2003b. Olfactory sensory system for odour-plume tracking and localization. In IEEE Int. Conf. on Sensors.

Marques, L., Nunes, U., and de Almeida, A. 2002a. Cooperative odour field exploration with genetic algorithms. In Proc. 5th Portuguese Conf. on Automatic Control (CONTROLO 2002), pp. 138-143.

Marques, L., Nunes, U., and de Almeida, A. 2002b. Olfaction-based mobile robot navigation. Thin Solid Films, 418(1):51-58.

Marques, L., Nunes, U., and de Almeida, A. 2003c. Odour searching with autonomous mobile robots: An evolutionary-based approach. In Proc. IEEE Int. Conf. on Advanced Robotics, pp. 494-500.

Müeller, S., Marchetto, J., Airaghi, S., and Koumoutsakos, P. 2002. Optimization based on bacterial chemotaxis. IEEE Trans. on Evolutionary Computation, 6(1):16-29.

Mole, N. and Jones, C. 1994. Concentration fluctuation data from dispersion experiments carried out in stable and unstable conditions. Boundary-Layer Meteorol, 67:41-74.

Mylne, K.R. and Mason, P.J. 1991. Concentration fluctuation measurements in a dispersing plume at a range of up to $1000 \mathrm{~m}$. Quart Journal Royal Meteorological Soc, 117:177-206.

Nielsen, M., Chatwin, P., Jørgensen, H.E., Mole, N., Munro, R., and Ott, S. 2002. Concentration fluctuations in gas releases by industrial accidents - Final report. Technical Report R-1329(EN), Risø Nat. Lab., Denmark.

Parsopoulos, K. and Vrahatis, M. 2002. Recent approaches to global optimization problems through particle swarm optimization. Natural Computing, 1(2-3):235-306.

Passino, K. 2002. Biomimicry of bacterial foraging for distributed optimization and control. IEEE Control Systems Magazine, 22(3):52-67.

Russell, R., Thiel, D., Deveza, R., and Mackay-Sim, A. 1995. A robotic system to locate hazardous chemical leaks. In Proc. IEEE Int. Conf. on Robotics and Automation, pp. 556-561.

Rutkowski, A., Edwards, S., Willis, M., and Quinn, R. 2004. A robotic platform for testing moth-inspired plume tracking strategies. In Proc. IEEE Int. Conf. on Robotics and Automation, pp. 3319-3324.

Stix, G. 2005. Better than a dog: The search is on for a sensor that bests a canine at detecting explosives. Scientific American Mag, 293(4):74-77. 
Stone, J. 1989. Theory of Optimal Search. Academic Press, 2nd edn. Yang, C. 2005. The state of surveillance. Business Week, pp. 52-59.

Yee, E., Chan, R., Kosteniuk, P., Chandler, G., Biltoft, C., and Bowers, J. 1994. Experimental measurements of concentration and scales in a dispersing plume in the atmospheric surface layer obtained using a very fast response concentration detector. Journal App. Meteorology, 33(8):996-1016.

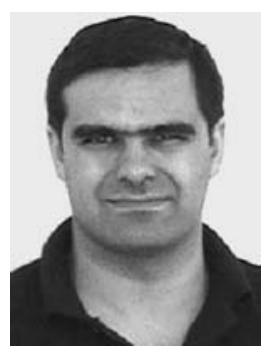

Lino Marques is an auxiliary professor in the Department of Electrical Engineering, University of Coimbra, and he is a researcher in the Institute for Systems and Robotics (ISR-UC). He received his Licenciatura, MSc. and $\mathrm{Ph} . \mathrm{D}$. degrees in Electrical Engineering from the University of Coimbra, Portugal. His main research interests include embedded systems, mechatronics, robotics for risky environments, optical range sensors, artificial olfaction systems and mobile robot olfaction.

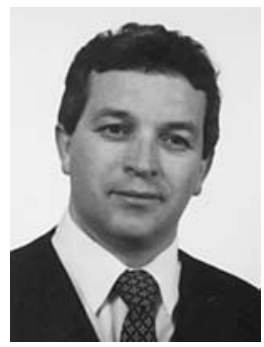

Urbano Nunes is an associate professor of the University of Coimbra and a researcher of the Institute for Systems and Robotics (ISR$\mathrm{UC})$, where he has been involved in research and teaching since 1983 . He received his Licenciatura and Ph.D. degrees in Electrical Engineering from the University of Coimbra, Portugal, in 1983 and 1995, respectively. He is the coordinator of the Mechatronics Laboratory of ISR-UC, and had been responsible for several funded projects in the areas of mobile robotics and intelligent vehicles. His research interests include mobile robotics, intelligent vehicles, and mechatronics. Professor Urbano Nunes serves on the Editorial Board of the Journal on Machine Intelligence and Robotic Control, and currently he is co-chair of the IEEE RAS TC on Intelligent Transportation Systems. Currently he is the Program Chair of the IEEE ITSC2006. He has served as General Co-Chair of ICAR 2003 and as member of several program committees of international conferences.

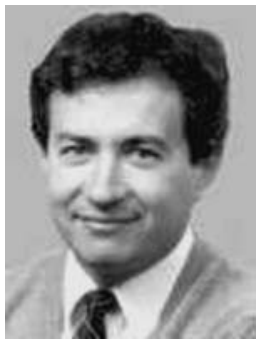

Aníbal T. De Almeida graduated in Electrical Engineering, University of Porto, 1972, and received a Ph.D. in Electrical Engineering, from Imperial College, University of London, 1977. Currently he is a Professor in the Department of Electrical Engineering, University of Coimbra, and he is the Director of the Institute of Systems and Robotics since 1993. Professor De Almeida is a consultant of the European Commission Framework Programmes. He is the co-author of five books and more than one hundred papers in international journals, meetings and conferences. He has coordinated several European and national research projects. 